\title{
Cisplatin resistance-associated circRNA_101237 serves as a prognostic biomarker in hepatocellular carcinoma
}

\author{
SHUPING ZHOU ${ }^{1}$, JUAN WEI ${ }^{2}$, YELIU WANG ${ }^{1}$ and XINKUANG LIU ${ }^{1}$ \\ ${ }^{1}$ Department of Gastroenterology and Hepatology, Huainan First People's Hospital and First Affiliated Hospital of \\ The Medical College of Anhui University of Science and Technology, Huainan, Anhui 232000; \\ ${ }^{2}$ Department of Gastroenterology and Hepatology, Nanjing General Hospital, Nanjing, Jiangsu 210002, P.R. China
}

Received March 11, 2019; Accepted October 2, 2019

DOI: $10.3892 /$ etm.2020.8526

\begin{abstract}
Hepatocellular carcinoma (HCC) is a leading cause of cancer-associated mortality worldwide. Despite clinical advances, the survival rate of patients with HCC remains low, as most patients are diagnosed with $\mathrm{HCC}$ when they are already at the advanced stage. Certain circular RNAs (circRNAs) are closely associated with the development of liver cancer. In the present study, a circRNA array was performed to screen differentially expressed circRNAs in HCC tissues. The further analysis focused on the newly identified circRNA_101237, the host gene of which, cyclin-dependent kinase 8 , is located at chr13:26974589-26975761. CircRNA_101237 was determined to be upregulated in tumor tissue and serum of patients with HCC as compared with that in paracancerous tissues and the serum of healthy controls, respectively. In addition, the expression of circRNA_101237 was associated with tumor size, lymph node metastasis, distant metastasis and TNM stage. Univariate and multivariate analysis indicated that serum circRNA_101237 levels were an independent predictor of survival prognosis in patients with HCC. The overall survival of patients with high expression of circRNA_101237 was reduced compared with that of patients with low expression of circRNA_101237. Of note, cisplatin induced the expression of circRNA_101237 in HCC cells in a dose- and time-dependent manner in vitro, and the levels of circRNA_101237 in the serum of patients with cisplatin-resistant HCC and in cisplatin-resistant Huh7 cells were increased. The present study provided novel insight into the use of circRNA_101237 as a diagnostic biomarker for HCC and a potential therapeutic target.
\end{abstract}

Correspondence to: Professor Xinkuang Liu, Department of Gastroenterology and Hepatology, Huainan First People's Hospital and First Affiliated Hospital of The Medical College of Anhui University of Science and Technology, 202 Huaibing Road, Huainan, Anhui 232000, P.R. China

E-mail: liuaustar@163.com

Key words: hepatocellular carcinoma, circular RNA, prognosis, cisplatin resistance, circRNA_101237

\section{Introduction}

Hepatocellular carcinoma (HCC) is a leading cause of cancer-associated mortality worldwide. Although the clinical application of sorafenib, regorafenib and nivolumab has achieved significant progress in the treatment of $\mathrm{HCC}$, the survival rate of patients with HCC remains low, as most patients with $\mathrm{HCC}$ are diagnosed at an advanced stage (1). Cisplatin and various platinum agents have become standard drugs for the treatment of HCC (2). However, as cisplatin resistance may occur, the survival benefit for patients with advanced HCC is unsatisfactory (3). To improve the survival of patients with HCC, novel biomarkers for early diagnosis and the development of novel therapeutic targets for cisplatin-resistant HCC are required (4).

Liver cancer is associated with hepatitis B virus (HBV), $\mathrm{HCV}$ and non-alcoholic fatty liver disease (5). However, the molecular mechanisms of the genesis of HCC remain poorly understood. Recently, new evidence has demonstrated that different types of non-coding RNAs (ncRNAs), including microRNAs (miRNAs), long ncRNAs and partially circular RNAs (circRNAs), have a role in HCC (6). High-throughput next-generation sequencing analysis has identified a large number of circRNAs that are involved in liver cancer through interactions with miRNAs or proteins $(7,8)$.

CircRNAs are generated from backsplicing of exons and introns, forming a circular exonic circRNA, a circular intronic RNA and an exon-intron circRNA. CircRNAs have a covalently closed continuous loop structure. They cannot be degraded by RNA exonuclease or RNase R. Therefore, circRNAs are suitable as diagnostic biomarkers for tumors, including HCC (9). CircRNAs are abundantly expressed in tissues, blood and microvesicles and are highly conserved among different species. CircRNAs are able to regulate gene expression at the transcriptional or post-transcriptional level (10). CircRNA is involved in various biological processes, including HCC cell proliferation, apoptosis and metastasis (11). For instance, overexpression of Homo sapiens circRNA_0001649 inhibits the proliferation and invasion of HCC cells (12). CircRNA circMTO1 (mitochondrial tRNA translation optimization 1) inhibits HCC progression by acting as a sponge for miRNA-9. Reduced levels of circMTO1 in HCC tissue may be used as a predictor of poor survival (13). 
In the present study, a circRNA array was performed to screen for differentially expressed circRNAs in HCC tissues. The study then focused on the newly identified circRNA_101237, whose encoding gene is located at chromosome (chr)13:26974589-26975761 and which is produced by backsplicing of exons 10,11 and 12 of cyclin-dependent kinase (CDK)8 (Fig. 1). CircRNA_101237 is upregulated in tumor tissues and serum of patients with $\mathrm{HCC}$ compared with that in paracancerous tissues and serum of healthy controls, respectively. In addition, the association between serum circRNA_101237 levels and the clinical outcome in patients with HCC was assessed.

\section{Materials and methods}

Patients and samples. A total of $100 \mathrm{HCC}$ cancer tissues and matched adjacent tissues were collected from Huainan First People's Hospital and the First Affiliated Hospital of the Medical College of Anhui University of Science and Technology (Huainan, China) between September 2013 and September 2017. The serum samples from another independent cohort, including 120 healthy individuals who came for physical examination and 234 patients with $\mathrm{HCC}$ were also collected from Huainan First People's Hospital and the First Affiliated Hospital of the Medical College of Anhui University of Science and Technology between September 2013 and September 2017. The general demographic and clinicopathological characteristics of 234 patients with HCC are shown in Table SI. The medical records of HCC patients with clinical TNM staging and survival information were collected. Patients with cisplatin-resistant $\mathrm{HCC}$ were defined as those with persistent disease at $>6$ weeks and those with recurrent disease at $>2$ months after completion of cisplatin-based chemotherapy. Patients with cisplatin-sensitive HCC were defined as those without local residual lesions at 6 weeks or no recurrence at 2 months after completion of cisplatin-based chemotherapy. The cisplatin-based chemotherapy regimen consisted of doxorubicin $60 \mathrm{mg} / \mathrm{m}^{2}$, followed by cisplatin $60 \mathrm{mg} / \mathrm{m}^{2}$ infused over $30 \mathrm{~min}$ on day 1 . Chemotherapy cycles were repeated every 21 days for 3 cycles (14).

Cell culture and treatment. The HCC cell lines HCCLM3, Hep3B and MHCC97-H were obtained from the Cell Bank of Type Culture Collection of the Chinese Academy of Sciences. All of the cells were grown routinely in RPMI-1640 medium (Invitrogen; Thermo Fisher Scientific, Inc.) supplemented with $10 \%$ fetal bovine serum (Gibco; Thermo Fisher Scientific, Inc.) and cultured at $37^{\circ} \mathrm{C}$ in a humidified atmosphere with $5 \% \mathrm{CO}_{2}$.

The HCC cell lines were exposed to cisplatin at $0,0.5$, 1 and $2 \mu \mathrm{g} / \mathrm{ml}$ for $48 \mathrm{~h}$ or at $1 \mu \mathrm{g} / \mathrm{ml}$ for $0,12,24$ or $48 \mathrm{~h}$, and the expression of circRNA_101237 was then assessed by reverse transcription-quantitative (RT-q) PCR.

Cisplatin-resistant cells. Parental Huh7 cells were obtained from The Cell Bank of Type Culture Collection of the Chinese Academy of Sciences and cultured in DMEM medium containing 10\% fetal bovine serum (Gibco; Thermo Fisher Scientific, Inc.). The cisplatin-resistant cells (Huh7/DDP) were established as previously described (15). Briefly, Huh7 cells were treated with a low concentration $(10 \mathrm{ng} / \mathrm{ml})$ of cisplatin (Sigma-Aldrich; Merck KGaA) for $72 \mathrm{~h}$. The medium containing $10 \mathrm{ng} / \mathrm{ml}$ of cisplatin was refreshed every 3 days for a total of 5 times. Further resistance was established by gradually raising the concentration of cisplatin in the culture solution until a target resistance of $5 \mu \mathrm{g} / \mathrm{ml}$ cisplatin was acquired.

CircRNA array. A total of 3 HCC tissues and the matched adjacent tissue samples were randomly selected for circRNA microarray. The circRNA microarray data were analyzed using Arraystar Human circRNA Array V2 analysis (Arraystar) by Kangchen BioTech Inc. In brief, total RNAs were digested with RNase R to remove linear RNAs and enrich circular RNAs. The enriched circular RNAs were amplified and transcribed into fluorescent circRNA utilizing a random priming method (Arraystar Super RNA Labeling Kit; Arraystar). The labeled circRNAs were hybridized onto the Arraystar Human circRNA Array V2 (8x15K; Arraystar). Following washing of the slides, the arrays were scanned by the Agilent Scanner G2505C. Agilent Feature Extraction software (version 11.0.1.1) was used to analyze the acquired array images. Differentially expressed circRNAs were then identified by analyzing the fold change, as well as the P-value. The threshold for significantly up- and downregulated genes was set as fold change $>2.0$ and $\mathrm{P}<0.05$.

$R T-q P C R$ analysis. Total RNA was extracted with TRIzol reagent (Invitrogen; Thermo Fisher Scientific, Inc.) and reverse transcribed to complementary DNA by using SuperScript $^{\text {TM }}$ IV VILO ${ }^{\text {TM }}$ Master Mix with ezDNase ${ }^{\mathrm{TM}}$ Enzyme (cat. no. 11766050; Thermo Fisher Scientific, Inc.) according to the manufacturer's protocol. PCR amplification was then performed with TaqMan Fast Advanced Master Mix (cat. no. 4444558; Thermo Fisher Scientific, Inc.) according to the manufacturers' protocol. $\beta$-actin expression was assessed as an endogenous control. qPCR was performed using the following conditions: $50^{\circ} \mathrm{C}$ for $2 \mathrm{~min}, 95^{\circ} \mathrm{C}$ for $2 \mathrm{~min}$ and 40 circles of $95.0^{\circ} \mathrm{C}$ for $1 \mathrm{sec}$ and $60^{\circ} \mathrm{C}$ for $20 \mathrm{sec}$. The primers used were as follows: circRNA_101237 forward, 5'-TGAGCT TGTGAGTGAGTGGT-3' and reverse, 5'-GCAAGGAGAATG GCGAGATG-3'; $\beta$-actin forward, 5'-TTGTTACAGGAAGTC CCTTGCC-3' and reverse, 5'-ATGCTATCACCTCCCCTG TGTG-3'. The $2^{-\Delta \Delta C a}$ method was used to analyze the qPCR data (16).

Statistical analysis. Experiments were performed as three independent replicates. Values are expressed as the mean \pm standard deviation and statistical analysis was performed using SPSS 17.0 statistical software (SPSS, Inc.). Differences among the groups were estimated by Student's t-test or one-way analysis of variance with Tukey's post-hoc test. The cases of HCC were divided into a high circRNA_101237 expression group (expression above the mean value) or otherwise into a low circRNA_100053 expression group. Good prognosis of patients with $\mathrm{HCC}$ was defined as a five-year overall survival probability of $\geq 60 \%$, while a lower probability was defined as poor prognosis. The overall survival rate estimates over time were calculated using the Kaplan-Meier method with log-rank tests. The association 


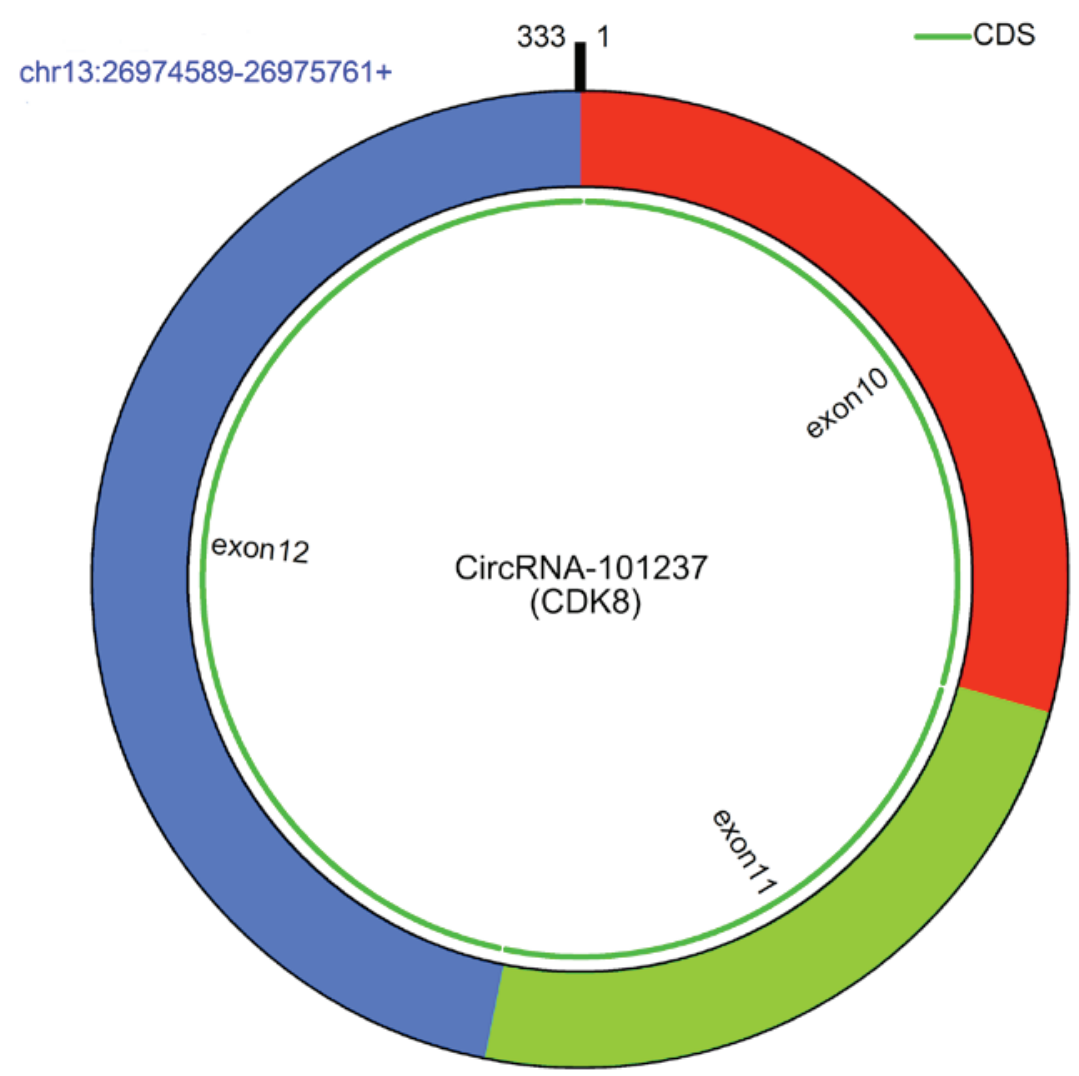

Figure 1. Schematic depicting the composition of circRNA_101237 (encoded by CDK8). circRNA, circular RNA; CDK8, cyclin-dependent kinase 8; chr, chromosome.

between circRNA_101237 expression and clinicopathological variables of HCC patients was evaluated using the Chi-squared test. Univariate and multivariate logistic regression analyses using the Cox proportional hazards model were performed to analyze prognostic factors. $\mathrm{P}<0.05$ was considered to indicate statistical significance.

\section{Results}

CircRNA_101237 is upregulated in tumor tissues and peripheral blood serum from patients with HCC. To investigate the role of circRNAs in HCC, circRNA microarray was performed to screen differentially expressed circRNAs. Upregulation was seen in 65 circRNAs and downregulation was seen in 87 circRNAs in HCC tissues vs. adjacent controls. As indicated in the heatmap, circRNA_101237 was significantly upregulated in HCC tissues compared with that in the adjacent controls (Fig. 2A). The expression of circRNA_101237 was further confirmed in 100 HCC tissues and the adjacent tissues. The quantitative results suggested that circRNA_101237 was significantly increased in HCC tissues compared with that in the adjacent tissues (Fig. 2B). To further investigate whether circRNA_101237 in the peripheral blood may serve as a diagnostic biomarker for HCC, circRNA_101237 expression was measured in an independent cohort, including serum samples from 234 patients with HCC and 120 healthy controls. CircRNA_101237 was also significantly upregulated in serum samples from patients with HCC compared with those from healthy controls (Fig. 2C).
High circRNA_101237 expression is associated with poor outcome for patients with HCC. The association between circRNA_101237 expression and clinicopathological features of HCC patients was then analyzed. The HCC patients were stratified into a high circRNA_101237 group and low circRNA_101237 group based on the mean level of circRNA_101237. The results indicated that the expression of circRNA_101237 was associated with tumor size $(\mathrm{P}<0.001)$, lymph node metastasis $(\mathrm{P}=0.006)$, distant metastasis $(\mathrm{P}=0.0002)$, TNM stage $(\mathrm{P}=0.0002)$ and Barcelona Clinic Liver Cancer (BCLC) stage $(\mathrm{P}<0.001$; Table I). In addition, univariate analysis suggested that the serum levels of circRNA_101237 (hazard ratio $=3.29, \mathrm{P}=0.01$ ), as well as the tumor size (hazard ratio=3.24, $\mathrm{P}=0.01$ ), lymph node metastasis (hazard ratio $=2.76, \mathrm{P}=0.03$ ), distant metastasis (hazard ratio $=5.72, \mathrm{P}=0.01$ ), $\mathrm{BCLC}$ stage (hazard ratio $=2.87$, $\mathrm{P}=0.02$ ) and $\mathrm{TNM}$ stage (hazard ratio=4.15, $\mathrm{P}=0.03$ ) were significantly associated with the prognosis of patients with HCC (Table II). Multivariate analysis revealed that the serum levels of circRNA_101237 (hazard ratio=3.42, $\mathrm{P}=0.02$ ), tumor size (hazard ratio $=3.14, \mathrm{P}=0.03$ ), lymph node metastasis (hazard ratio $=3.76, \mathrm{P}=0.02$ ), distant metastasis (hazard ratio $=4.35, \mathrm{P}=0.01$ ), $\mathrm{BCLC}$ stage (hazard ratio $=3.25$, $\mathrm{P}=0.03$ ) and TNM stage (hazard ratio=3.93, $\mathrm{P}=0.03$ ) were independent prognostic factors for the survival of patients with HCC (Table III). The association between serum levels of circRNA_101237 in HCC patients and overall survival was then further analyzed. The Kaplan-Meier survival curves indicated that the patients with high circRNA_101237 expression had a significantly poorer 
A

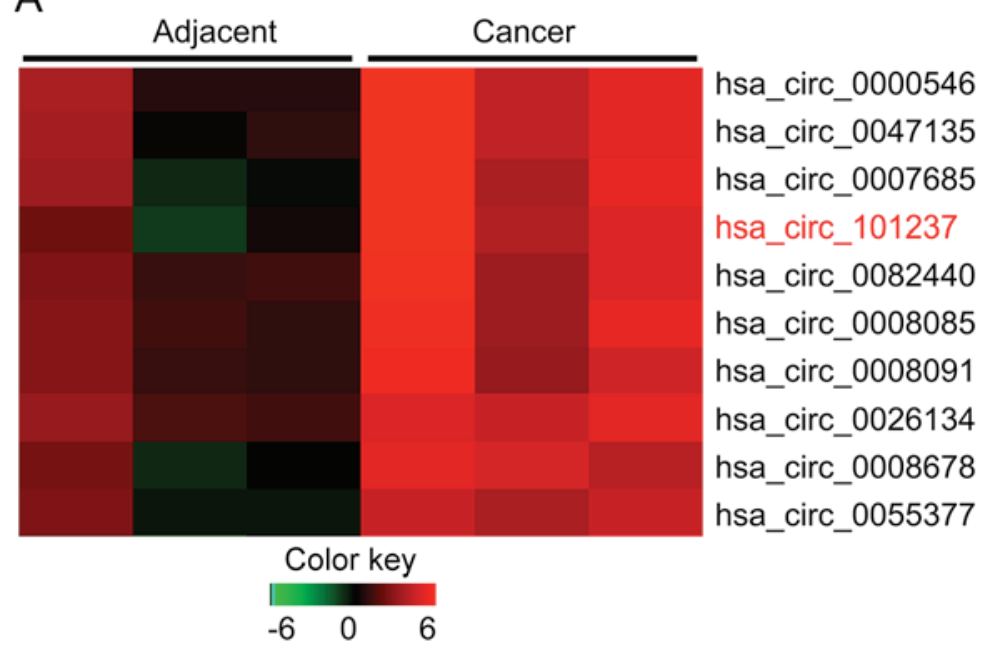

B

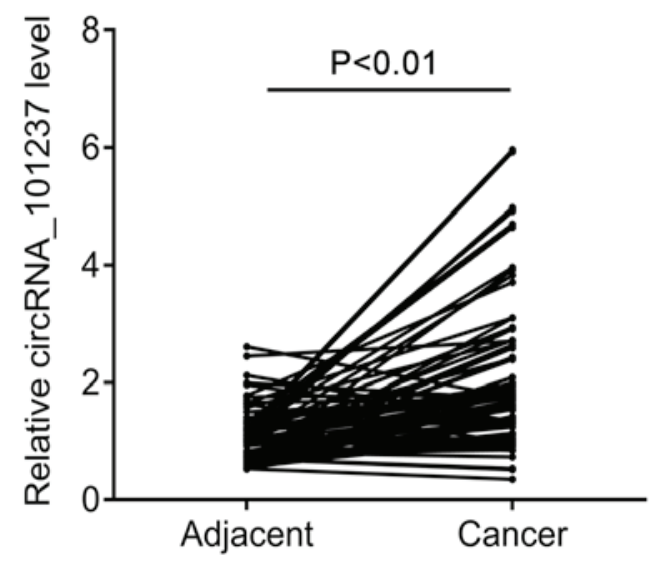

D

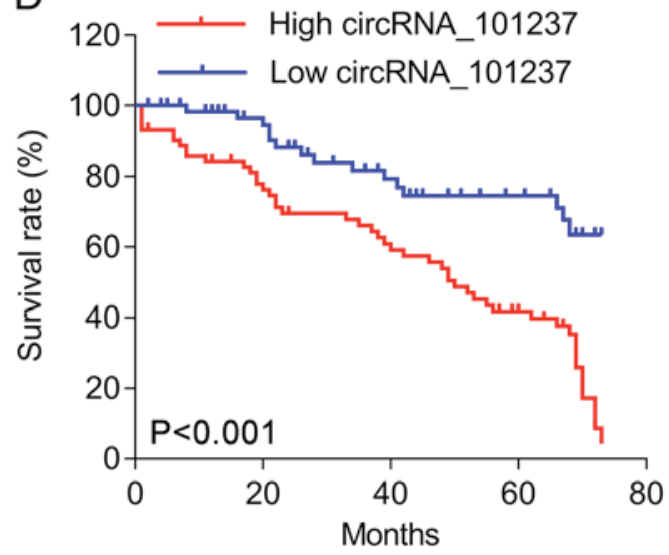

Figure 2. CircRNA_101237 expression in HCC tissues. (A) Heatmap for differentially expressed circRNAs in HCC tumor tissues and the matched adjacent tissues $(n=3)$. (B) RT-qPCR was performed to determine the expression of circRNA_101237 in tumor tissues ( $\mathrm{n}=100)$ and adjacent tissues ( $\mathrm{n}=100)$. (C) RT-qPCR was performed to assess the expression of circRNA_101237 in serum samples from patients with HCC (n=234) and healthy controls ( $\mathrm{n}=120$ ). (D) Kaplan-Meier survival curves were drawn to compare the survival of patients with high circRNA_101237 expression (n=148) and those with low circRNA_101237 expression (n=86). RT-qPCR, reverse transcription-quantitative PCR; HCC, hepatocellular carcinoma; circRNA_101237, circular RNA 101237; hsa, Homo sapiens.

overall survival than those with low circRNA_101237 expression $(\mathrm{P}<0.001$; Fig. 2D). Overall, the results suggest that circRNA_101237 expression has a negative impact on the prognosis of patients with HCC.

Circ_101237 expression is associated with cisplatin resistance in patients with HCC. Of note, circRNA_101237 was increased by $\sim 3$-fold in the serum of cisplatin-resistant HCC patients $(n=50)$ compared with that in cisplatin-sensitive patients $(n=62)$ (Fig. 3A). In addition, the expression of circRNA_101237 in liver cancer cell lines was determined, and the results indicated that circRNA_101237 was significantly upregulated in the MHCC97-H and Huh7 cell lines as compared with that in HCCLM3 cells (Fig. 3B). Of note, circRNA_101237 levels in cisplatin-resistant Huh7/DDP cells was higher than that in the parental cells (Fig. 3B). In addition, HCCLM3, Hep3B, MHCC97-H and Huh7 cells were confirmed to respond to cisplatin by upregulating circRNA_101237 in a time- and cisplatin dose-dependent manner (Fig. 4). These results suggest that circRNA_101237 may be used as a biomarker for HCC diagnosis and cisplatin resistance in patients with $\mathrm{HCC}$.

\section{Discussion}

In the present study, a novel circRNA, circRNA_101237, was identified as a diagnostic biomarker for HCC and a potential therapeutic target. CircRNA_101237 was upregulated in HCC tumor tissues compared with that in adjacent tissues. It was further confirmed that circRNA_101237 is upregulated in serum samples from patients with HCC. The upregulation of circRNA_101237 was positively associated with tumor size, lymph node metastasis, distant metastasis and TNM stage of HCC patients. In addition, univariate and multivariate analysis suggested that circRNA_101237 is an independent predictor of prognosis in patients with HCC. These results indicate that circRNA_101237 has a key role in the development of HCC.

If cancer is diagnosed at an early stage, the 5-year survival rate of HCC patients is better (probably $>70 \%$ ) (17). 
Table I. Clinical association between serum circRNA_101237 levels and clinicopathological characteristics of patients with hepatocellular carcinoma.

Serum circRNA_101237

Variable $\quad$ Low expression $(\mathrm{n}=86) \quad$ High expression $(\mathrm{n}=148) \quad \chi^{2}$ test P-value

Age (years)

$<50$

56

0.4070

$\geq 50$

30

88

60

Sex

Male

Female

Tumor size $(\mathrm{cm})$

$<0.001$

$<3$

60

48

$\geq 3$

26

100

Lymph node metastasis

0.0060

N0-1

61

N2-4

Distant metastasis

TNM stage

I-II

III-IV

BCLC stage

\section{0 or $\mathrm{A}$}

$\mathrm{B}$

C

D

ALBI grade

1

2

3

Diabetes mellitus

No

Yes

Body mass index $\left(\mathrm{kg} / \mathrm{m}^{2}\right)$

$<30$

$\geq 30$
32

26

20

8

34

26

26

72

14

54

32
0.0002

55

93

$<0.001$

13

34

56

45

0.089

42

65

41

0.147

112

36

0.48

86

62

BCLC, Barcelona Clinic Liver Cancer; ALBI, albumin-bilirubin; circRNA_101237, circular RNA 101237.

Early diagnosis of $\mathrm{HCC}$ is difficult due to inflammation and cirrhosis. Therefore, there is an urgent requirement to develop novel biomarkers for early diagnosis of HCC (11). Recently, serum alpha-fetoprotein, phosphatidylinositol-3, osteopontin, Golgi protein-73 and various miRNAs have been reported as promising early biomarkers of HCC, providing insight into the mechanisms that drive tumorigenesis, which may lead to the development of more effective treatment strategies (18). In the present study, circRNA_101237 was revealed as a novel biomarker for HCC. However, in the present cohort of HCC patients, no detailed information was available regarding HBV, HCV and non-alcoholic fatty liver disease. As the influence of hepatitis/NAFLD was unknown, it was not reasonable to analyze the association between circRNA_101237 and cirrhosis in patients with liver cancer. Furthermore, in the future, analysis of the correlation 
Table II. Univariate analysis of prognostic factors for patients with hepatocellular carcinoma.

\begin{tabular}{lcc}
\hline Variable & Hazard ratio $(95 \%$ confidence interval) & P-value \\
\hline Age $(\geq 50 /<50$ years $)$ & $1.06(0.870-1.142)$ & 0.17 \\
Sex (male/female) & $1.02(0.943-1.165)$ & 0.34 \\
Tumor size $(\geq 3 /<3 \mathrm{~cm})$ & $3.24(1.820-7.322)$ & 0.01 \\
Lymph node metastasis (N2-4/N0-1) & $2.76(1.346-5.276)$ & 0.03 \\
Distant metastasis $($ yes/no) & $5.72(2.703-9.352)$ & 0.01 \\
TNM stage (III-IV/I-II) & $4.15(1.492-7.626)$ & 0.03 \\
Serum circRNA_101237 levels (high/low) & $3.29(2.632-8.544)$ & 0.01 \\
BCLC stage (C-D/0-B) & $2.87(1.375-6.432)$ & 0.02 \\
ALBI grade (III/I-II) & $1.2(0.978-3.651)$ & 0.06 \\
Diabetes mellitus $(y e s / n o)$ & $1.08(0.774-1.692)$ & 0.17 \\
Body mass index $\left(\geq 30 /<30 \mathrm{~kg} / \mathrm{m}^{2}\right)$ & $1.03(0.641-1.863)$ & 0.36 \\
\hline
\end{tabular}

BCLC, Barcelona Clinic Liver Cancer; ALBI, albumin-bilirubin; circRNA_101237, circular RNA 101237.

Table III. Multivariate analysis of independent prognostic factors for patients with hepatocellular carcinoma.

\begin{tabular}{lcr}
\hline Variable & Hazard ratio (95\% confidence interval) & P-value \\
\hline Tumor size $(\geq 3 /<3 \mathrm{~cm})$ & $3.14(2.522-5.541)$ & 0.03 \\
Lymph node metastasis (N2-4/N0-1) & $3.76(1.765-8.547)$ & 0.02 \\
Distant metastasis (yes/no) & $4.35(3.431-8.651)$ & 0.01 \\
TNM stage (III-IV/I-II) & $3.93(2.086-6.322)$ & 0.03 \\
BCLC stage (C-D/0-B) & $3.25(2.268-8.634)$ & 0.03 \\
Serum circRNA_101237 levels (high/low) & $3.42(2.215-6.532)$ & 0.02 \\
\hline
\end{tabular}

BCLC, Barcelona Clinic Liver Cancer; ALBI, albumin-bilirubin.

between circRNA_101237 and recently used biomarkers may provide meaningful information for early diagnosis of HCC. These points will be further investigated in future studies by our group.

The present results indicated that circRNA_101237 levels were increased in cisplatin-resistant HCC tumor tissues and cisplatin-resistant Huh7 cells, and cisplatin exposure induced an increase in circRNA_101237 expression, suggesting that circRNA_101237 may be a biomarker of cisplatin resistance in patients with HCC. Cisplatin has been widely used to treat a variety of cancer types, including HCC (19). Patients with HCC initially respond to cisplatin therapy but resistance frequently occurs, which is associated with increased DNA repair, altered cell accumulation and increased drug efflux mediated by multidrug resistance proteins (20). It has been reported that cisplatin resistance in HCC may occur through the loss of Runt-associated transcription factor 3 and upregulation of cyclophilin B $(21,22)$. Recently, certain key biomarkers of cisplatin resistance have revealed novel molecular mechanisms of resistance (23). The role of circRNAs in the development of chemotherapeutic drug resistance has also been highlighted (24-26). For instance, hsa_circ_0004674 is increased in chemoresistant osteosarcoma cells and tissues and is associated with poor prognosis (27). Upregulation of circRNA-MTO1 promotes monastrol-induced cytotoxicity and reverses monastrol resistance by inhibiting Eg5 (28). CircRNA-PVT1 (plasmocytoma variant translocation) is significantly upregulated in osteosarcoma, serum and chemoresistant cell lines, including those with doxorubicin and cisplatin resistance. CircRNA-PVT1 knockdown overcomes the resistance of osteosarcoma cells to doxorubicin and cisplatin (29). The present study indicated that patients with cisplatin-resistant HCC and cisplatin-resistant Huh7/DDP cells had increased levels of circRNA_101237, but it remains elusive whether circRNA_101237 knockdown is able to inhibit HCC cell proliferation and sensitize HCC cells to cisplatin. These biological roles of circRNA_101237 in HCC cells will be demonstrated in the future and the underlying mechanisms will be revealed.

In conclusion, the present study indicated that circRNA_101237 is upregulated in tissues and serum of patients with HCC and may serve as a diagnostic and prognostic biomarker. In addition, the levels of circRNA_101237 were increased in the serum of cisplatin-resistant HCC patients and in cisplatin-resistant Huh7 cells. The present 
A

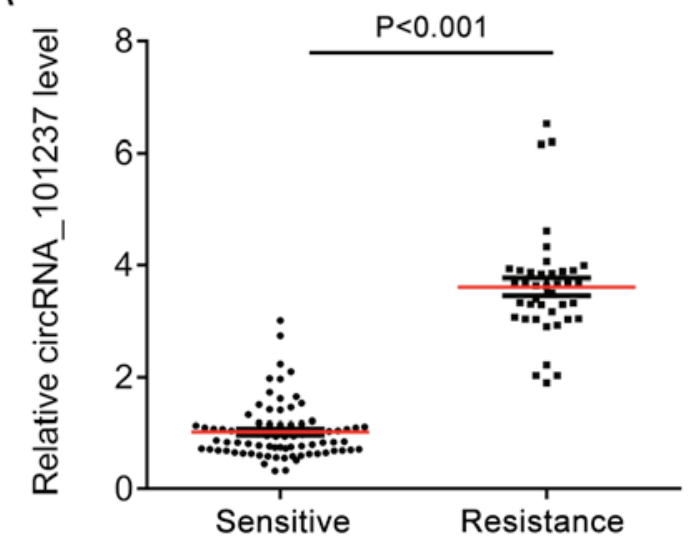

B

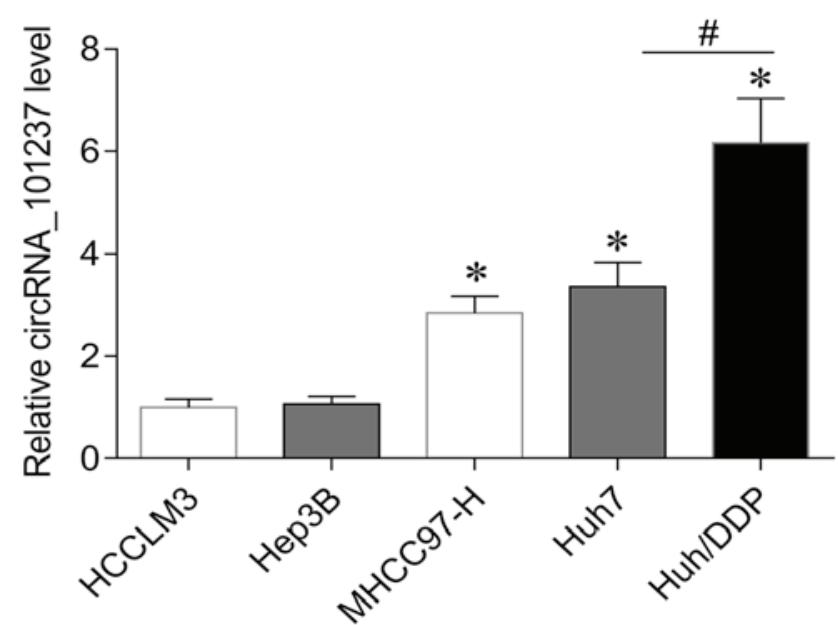

Figure 3. Expression of circRNA_101237 is associated with cisplatin resistance. (A) RT-qPCR was performed to assess the expression of circRNA_101237 in serum samples of patients with cisplatin-sensitive HCC $(n=50)$ and those with cisplatin-resistant HCC $(n=35)$. (B) RT-qPCR was performed to determine the expression of circRNA_101237 in liver cancer cells. " $\mathrm{P}<0.05$ vs. HCCLM3 cells; ${ }^{\# P} \mathrm{P}<0.05$, Huh7/DDP vs. Huh7. Huh/DDP, cisplatin-resistant Huh7 cells; RT-qPCR, reverse transcription-quantitative PCR; HCC, hepatocellular carcinoma; circRNA_101237, circular RNA 101237.

A Cisplatin treatment $(48 \mathrm{~h})$

$$
\begin{aligned}
& 0 \\
& 0.5 \mu \mathrm{g} / \mathrm{ml}=1 \mu \mathrm{g} / \mathrm{ml} \\
& 2 \mu \mathrm{g} / \mathrm{ml}
\end{aligned}
$$

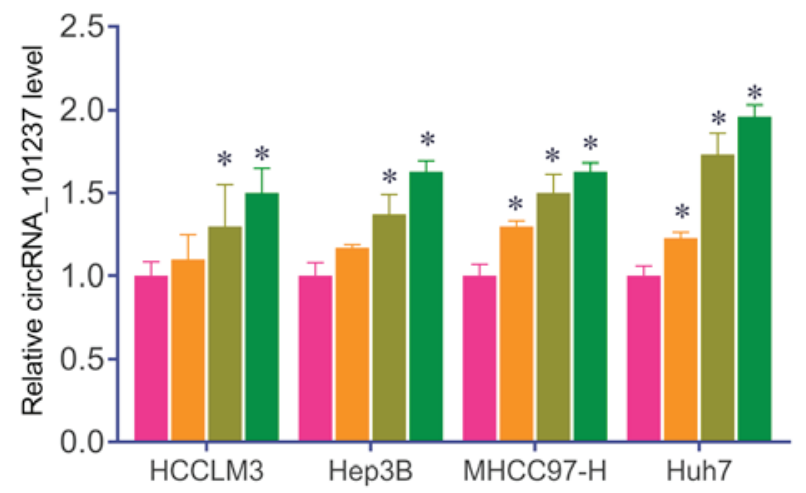

B Time of cisplatin treatment $(1 \mu \mathrm{g} / \mathrm{ml})$

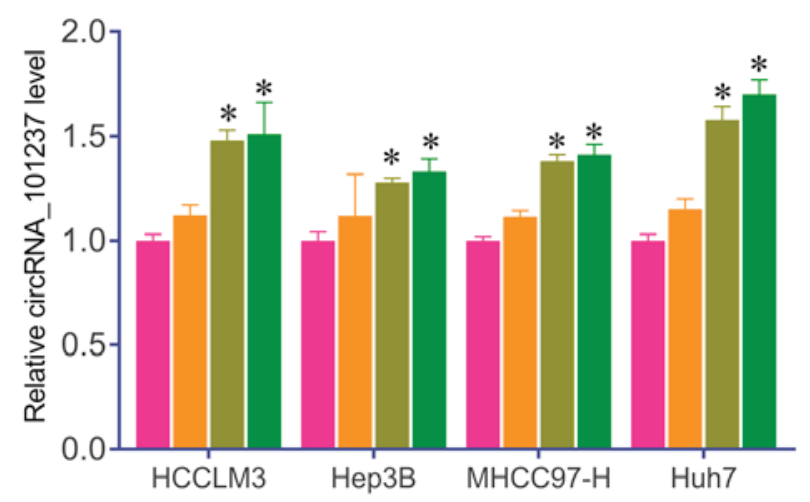

Figure 4. Changes in circRNA_101237 expression in hepatocellular carcinoma cell lines in response to cisplatin treatment. (A) Cells were treated for $48 \mathrm{~h}$ with cisplatin at $0,0.5,1$ and $2 \mu \mathrm{g} / \mathrm{ml}$. (B) Cells were treated with cisplatin at $1 \mu \mathrm{g} / \mathrm{ml}$ for $0,12,24 \mathrm{or} 48 \mathrm{~h}$. Reverse transcription-quantitative PCR was performed to assess the expression of circRNA_101237. "P<0.05 vs. controls. circRNA_101237, circular RNA 101237.

results provide evidence that circRNA_101237 may be used as a diagnostic biomarker for $\mathrm{HCC}$ and a potential therapeutic target for overcoming cisplatin resistance.

\section{Acknowledgements}

Not applicable.

\section{Funding}

No funding was received.

\section{Availability of data and materials}

The datasets used and/or analyzed during the current study are available from the corresponding author on reasonable request.

\section{Authors' contributions}

XL made substantial contributions to the design of the study. SZ, JW and YW analyzed and interpreted the patient data. SZ and JW performed the cell biological experiments. All authors contributed to the writing of the manuscript. All authors read and approved the final manuscript.

\section{Ethics approval and consent to participate}

The present study was approved by the Ethics Committee of Huainan First People's Hospital and the First Affiliated Hospital of the Medical College of Anhui University of Science and Technology (Huainan, China). All subjects provided written informed consent to participate in the present study. 


\section{Patient consent for publication}

Not applicable.

\section{Competing interests}

The authors declare that they have no competing interests.

\section{References}

1. Sia D, Villanueva A, Friedman SL and Llovet JM: Liver cancer cell of origin, molecular class, and effects on patient prognosis Gastroenterology 152: 745-761, 2017.

2. Ikeda K: Recent advances in medical management of hepatocellular carcinoma. Hepatol Res 49: 14-32, 2019.

3. Kawaoka T, Aikata H, Kobayashi T, Uchikawa S, Ohya K, Kodama K, Nishida Y, Daijo K, Osawa M, Teraoka Y, et al: Comparison of hepatic arterial infusion chemotherapy between 5-fluorouracil-based continuous infusion chemotherapy and low-dose cisplatin monotherapy for advanced hepatocellular carcinoma. Hepatol Res 48: 1118-1130, 2018.

4. Shang X,Li G,Liu H,LiT,Liu J,Zhao Q and Wang C: Comprehensive circular RNA profiling reveals that hsa_circ_0005075, a new circular RNA biomarker, is involved in hepatocellular carcinoma development. Medicine (Baltimore) 95: e3811, 2016.

5. Sun X and Malhotra A: Noncoding RNAs (ncRNA) in hepato cancer: A review. J Environ Pathol Toxicol Oncol 37: 15-25, 2018

6. Lanzafame M, Bianco G, Terracciano LM, Ng C and Piscuoglio S: The role of long non-coding RNAs in hepatocarcinogenesis. Int J Mol Sci 19: E682, 2018

7. Qiu LP, Wu YH, Yu XF, Tang Q, Chen L and Chen KP: The emerging role of circular RNAs in hepatocellular carcinoma. J Cancer 9: 1548-1559, 2018.

8. Liu BH, Zhang BB, Liu XQ, Zheng S, Dong KR and Dong R: Expression profiling identifies circular RNA signature in hepatoblastoma. Cell Physiol Biochem 45: 706-719, 2018.

9. Wang M, Yang Y, Xu J, Bai W, Ren X and Wu H: CircRNAs as biomarkers of cancer: A meta-analysis. BMC Cancer 18: 303, 2018

10. Zhu Q, Lu G, Luo Z, Gui F, Wu J, Zhang D and Ni Y: CircRNA circ_0067934 promotes tumor growth and metastasis in hepatocellular carcinoma through regulation of miR-1324/FZD5/Wnt/ $\beta$-catenin axis. Biochem Biophys Res Commun 497: 626-632,2018.

11. Shi L, Yan P, Liang Y, Sun Y, Shen J, Zhou S, Lin H, Liang X and Cai X: Circular RNA expression is suppressed by androgen receptor (AR)-regulated adenosine deaminase that acts on RNA (ADAR1) in human hepatocellular carcinoma. Cell Death Dis 8: e3171, 2017.

12. Zhang X, Qiu S, Luo P, Zhou H, Jing W, Liang C and Tu J: Down-regulation of hsa_circ_0001649 in hepatocellular carcinoma predicts a poor prognosis. Cancer Biomark 22: 135-142, 2018.

13. Han D, Li J, Wang H, Su X, Hou J, Gu Y, Qian C, Lin Y, Liu X, Huang M, et al: Circular RNA circMTO1 acts as the sponge of microRNA-9 to suppress hepatocellular carcinoma progression. Hepatology 66: 1151-1164, 2017.
14. Shao YY, Liang PC, Wu YM, Huang CC, Huang KW, Cheng JC, Hsu CH, Hsu C, Cheng AL and Lin ZZ: A pilot study of hepatic arterial infusion of chemotherapy for patients with advanced hepatocellular carcinoma who have failed anti-angiogenic therapy. Liver Int 33: 1413-1419, 2013.

15. Wakamatsu T, Nakahashi Y, Hachimine D, Seki T and Okazaki K: The combination of glycyrrhizin and lamivudine can reverse the cisplatin resistance in hepatocellular carcinoma cells through inhibition of multidrug resistance-associated proteins. Int J Oncol 31: 1465-1472, 2007.

16. Livak KJ and Schmittgen TD: Analysis of relative gene expression data using real-time quantitative PCR and the 2(-Delta Delta C(T)) method. Methods 25: 402-408, 2001.

17. Fu J and Wang $\mathrm{H}$ : Precision diagnosis and treatment of liver cancer in China. Cancer Lett 412: 283-288, 2018.

18. Tsuchiya N, Sawada Y, Endo I, Saito K, Uemura Y and Nakatsura T: Biomarkers for the early diagnosis of hepatocellular carcinoma. World J Gastroenterol 21: 10573-10583, 2015.

19. Amable L: Cisplatin resistance and opportunities for precision medicine. Pharmacol Res 106: 27-36, 2016.

20. Hu Y, Zhu QN, Deng JL, Li ZX, Wang G and Zhu YS: Emerging role of long non-coding RNAs in cisplatin resistance. Onco Targets Ther 11: 3185-3194, 2018.

21. Kataoka J, Shiraha H, Horiguchi S, Sawahara H, Uchida D, Nagahara T, Iwamuro M, Morimoto H, Takeuchi $Y$, Kuwaki K, et al: Loss of Runt-related transcription factor 3 induces resistance to 5-fluorouracil and cisplatin in hepatocellular carcinoma. Oncol Rep 35: 2576-2582, 2016.

22. Kim Y, Jang M, Lim S, Won H, Yoon KS, Park JH, Kim HJ, Kim BH, Park WS, Ha J and Kim SS: Role of cyclophilin B in tumorigenesis and cisplatin resistance in hepatocellular carcinoma in humans. Hepatology 54: 1661-1678, 2011.

23. Achkar IW, Abdulrahman N, Al-Sulaiti H, Joseph JM, Uddin S and Mraiche F: Cisplatin based therapy: The role of the mitogen activated protein kinase signaling pathway. J Transl Med 16: 96 , 2018.

24. Xu N, Chen S, Liu Y, Li W, Liu Z, Bian X, Ling C and Jiang M Profiles and bioinformatics analysis of differentially expressed Circrnas in taxol-resistant non-small cell lung cancer cells. Cell Physiol Biochem 48: 2046-2060, 2018.

25. Ding B, Lou W, Xu L and Fan W: Non-coding RNA in drug resistance of hepatocellular carcinoma. Biosci Rep 38: BSR20180915, 2018.

26. Shao F, Huang M, Meng F and Huang Q: Circular RNA signature predicts gemcitabine resistance of pancreatic ductal adenocarcinoma. Front Pharmacol 9: 584, 2018.

27. Kun-Peng Z, Xiao-Long M, Lei Z, Chun-Lin Z, Jian-Ping H and Tai-Cheng Z: Screening circular RNA related to chemotherapeutic resistance in osteosarcoma by RNA sequencing. Epigenomics 10: 1327-1346, 2018.

28. Liu Y, Dong Y, Zhao L, Su L and Luo J: Circular RNAMTO1 suppresses breast cancer cell viability and reverses monastrol resistance through regulating the TRAF4/Eg5 axis. Int J Oncol 53 1752-1762, 2018.

29. Kun-Peng Z, Xiao-Long M and Chun-Lin Z: Overexpressed circPVT1, a potential new circular RNA biomarker, contributes to doxorubicin and cisplatin resistance of osteosarcoma cells by regulating ABCB1. Int J Biol Sci 14: 321-330, 2018. 